\title{
Stable circular orbits in higher-dimensional multi-black-hole spacetimes
}

\author{
Takahisa Igata $\odot^{1, *}$ and Shinya Tomizawa ${ }^{2, \dagger}$ \\ ${ }^{1}$ KEK Theory Center, Institute of Particle and Nuclear Studies, \\ High Energy Accelerator Research Organization, Tsukuba 305-0801, Japan \\ ${ }^{2}$ Mathematical Physics Laboratory, Toyota Technological Institute, Nagoya 468-8511, Japan
}

(Received 5 August 2020; accepted 2 September 2020; published 1 October 2020)

\begin{abstract}
We consider the dynamics of particles, particularly focusing on circular orbits in the higher-dimensional Majumdar-Papapetrou (MP) spacetimes with two equal mass black holes. It is widely known that in the 5D Schwarzschild-Tangherlini and Myers-Perry backgrounds, there are no stable circular orbits. In contrast, we show that in the 5D MP background, stable circular orbits can always exist when the separation of two black holes is large enough. More precisely, for a large separation, stable circular orbits exist from the vicinity of horizons to infinity; for a medium one, they appear only in a certain finite region bounded by the innermost stable circular orbit and the outermost stable circular orbit outside the horizons; for a small one, they do not appear at all. Moreover, we show that in MP spacetimes in more than 5D, they do not exist for any separations.
\end{abstract}

DOI: 10.1103/PhysRevD.102.084003

\section{INTRODUCTION}

The dynamics of test free particles in curved spacetimes, i.e., the geodesic structure, include important information about the gravitational field and the geometry. In stationary spacetimes, there can be stationary orbits of particles, which are geodesics along timelike Killing fields. Furthermore, if it is also axisymmetric, the stationary orbits can be circular orbits. Such fundamental orbits associated with spacetime symmetries are useful to understand various observable phenomena (e.g., the stellar motion and black hole shadow) around the black hole.

In the Schwarzschild black hole spacetime, there exist both stable and unstable circular orbits of particles. Let $r$ be the circumference radius, and let $M$ be the black hole mass. We know that the stable circular orbits exist in the range $r \geq 6 M$, and the unstable circular orbits in the range $3 M<r<6 M$, where we have used geometrized units. There exist the innermost stable circular orbit (ISCO) at their boundary $r=6 \mathrm{M}$ and the unstable photon circular orbit at the last circular orbit $r=3 M$. These are fundamentals of physical phenomena in the vicinity of a black hole. In the Kerr black hole spacetime, both stable and unstable circular orbits also appear [1].

\footnotetext{
igata@post.kek.jp

tomizawa@toyota-ti.ac.jp
}

Published by the American Physical Society under the terms of the Creative Commons Attribution 4.0 International license. Further distribution of this work must maintain attribution to the author(s) and the published article's title, journal citation, and DOI. Funded by SCOAP ${ }^{3}$.
In the last two decades, higher-dimensional black holes have also been actively studied [2], so that some of them are parametrized by the spacetime dimension $d$. The parametrization allows us to distinguish between $d$-dependent/ independent properties and also tells us some special properties in a specific $d$. Such dimensionality often appears in the analysis of gravitational properties through geodesic structure, which is the first step in the study of black holes. Unlike the 4D case, in a higher-dimensional static and spherically symmetric vacuum black hole, there is no stable circular orbit because no stable balance is formed between the gravitational and centrifugal forces [3], which is a generic feature of higher-dimensional black holes with a spherically symmetric horizon [4]. This property carries over to circular orbits in the 5D MyersPerry black holes [5-7] and equatorial circular orbits in the singly rotating Myers-Perry black holes in arbitrary dimensions [8]. Though it was pointed out that stable stationary/bound orbits can exist in the Myers-Perry black holes [9] at least when there is no upper limit on the black hole spin parameters (so-called the ultraspinning limit [10]), ${ }^{1}$ they tend not to appear for the higher-dimensional Myers-Perry family in general due to the dimensional dependence of the law of gravity.

However, there seems to be some exceptions to the nature that stable circular/bound orbits are less likely to appear under the diversity of higher-dimensional gravity. As one of the rich properties of higher-dimensional spacetimes, there is the topological variety of spatial cross

\footnotetext{
${ }^{1}$ In higher-dimensional AdS black holes, stable stationary orbits can appear because of the asymptotic structure [11,12].
} 
sections of horizons. In 5D asymptotically flat, stationary, and biaxisymmetric spacetimes, the allowed horizon topology is not only the sphere $S^{3}$ but also the ring $S^{1} \times S^{2}$ and the lens $L(p, q)$ [13-16]. We are gradually learning that the nontrivial horizon topologies of black objects can give rise to a mechanism for the appearance of stable stationary orbits that is different from the case of spherical black holes. In 5D black ring spacetimes [17], stable stationary orbits are absent in the fat regime, as is the spherical cases, but appears in the thin regime [18-21]. The existence of a nut ${ }^{2}$ outside the horizon plays an essential role in the existence of stable stationary orbits. Recently, even for the black rings in more than $5 \mathrm{D}$, the existence of stable stationary orbits and their dimensionality have been revealed using the blackfold approach [23,24]. In 5D black lens spacetimes [25,26], stable circular orbits can also exist [27]. Even in this phenomenon, it is also essential that the centers (i.e., the nuts) are located outside the horizon.

Concerning a higher-dimensional black hole with disconnected components of the horizon cross section, we encounter a nontrivial question of whether stable stationary/bound orbits exist and, if so, how they are distributed. This paper aims to clarify how the many-body nature of black holes in higher-dimensional spacetimes affects the existence of test particles' stationary orbits. To explore this, we adopt the two-body black hole configuration of the Majumdmar-Papapetrou (MP) geometry [28-30], which is kept static by balancing the gravitational and Coulomb force between the two with electric charges of the same sign. Since this family has singularities on the horizon but not outside it, we can examine the existence of the timelike/null geodesic Killing orbits and their stability throughout the domain of outer communication. The geodesic structure of the 4D MP dihole spacetime has been analyzed in detail in terms of circular/bound orbits and their stability [31-38], chaos [39,40], and shadows $[41,42]$. The particle dynamics in higher-dimensional MP spacetimes were investigated in Ref. [43]. Since the center is located outside the horizon in a higherdimensional two-body black hole spacetime, as well as the black rings and black lenses, we can expect the appearance of stable stationary orbits in the higherdimensional MP dihole spacetimes.

This paper is organized as follows. In Sec. II, we introduce the MP dihole spacetime in $d$ dimensions and formulate particle dynamics on the spacetime. Focusing specifically on stationary orbits, we identify the conditions for their existence and clarify criteria to determine whether they are stable. In Sec. III, for $d=5$, we clarify the dependence of the sequences of stationary orbits on the dihole separation parameter. In addition, based on these results, we give some critical values for the separation

\footnotetext{
${ }^{2}$ This terminology is often used to denote an isolated fixed point of one-parameter $U(1)$ isometry [22].
}

parameter. We discuss these properties for $d \geq 6$ as well. Section IV is devoted to a summary and discussions. Throughout this paper we use units in which $G=1$ and $c=1$.

\section{FORMULATION}

We focus on the MP geometries in $d$ dimensions $(d \geq 4)$. The metric and the gauge field are given by ${ }^{3}$

$$
\begin{gathered}
g_{\mu \nu} \mathrm{d} x^{\mu} \mathrm{d} x^{\nu}=-U^{-2} \mathrm{~d} t^{2}+U^{2 /(d-3)} \mathrm{d} \boldsymbol{r} \cdot \mathrm{d} \boldsymbol{r} \\
A_{\mu} \mathrm{d} x^{\mu}=\sqrt{\frac{d-2}{2(d-3)}} U^{-1} \mathrm{~d} t
\end{gathered}
$$

where $\mu, \nu$ are spacetime indices, and $\mathrm{d} \boldsymbol{r} \cdot \mathrm{d} \boldsymbol{r}$ is the $(d-1)$ dimensional flat metric, and $U$ is a harmonic function on $\mathbb{R}^{d-1}$ [28-30]. When $U$ has two point sources, the geometry represents a two-centered black hole spacetime. Using a $d$-dimensional cylindrical coordinate system $\left(z, \rho, \phi_{1}, \ldots, \phi_{d-3}\right)$ on $\mathbb{R}^{d-1}$, where $z$ is a cylindrical and Cartesian coordinate, $\rho$ is a radial coordinate from the $z$ axis, and $\phi_{a}(a=1, \ldots, d-3)$ are polar coordinates orthogonal to the $\rho-z$ plane, the metric of the MP dihole spacetime is given by

$g_{\mu \nu} \mathrm{d} x^{\mu} \mathrm{d} x^{\nu}=-U^{-2} \mathrm{~d} t^{2}+U^{2 /(d-3)}\left(\mathrm{d} z^{2}+\mathrm{d} \rho^{2}+\rho^{2} \mathrm{~d} \Omega_{d-3}^{2}\right)$,

$$
\begin{gathered}
U=1+\frac{M_{+}}{r_{+}^{d-3}}+\frac{M_{-}}{r_{-}^{d-3}}, \\
r_{ \pm}=\sqrt{(z \pm a)^{2}+\rho^{2}},
\end{gathered}
$$

where $M_{ \pm}$are masses of two extremal black holes placed at $z=\mp a$ on the $z$ axis, and $\mathrm{d} \Omega_{d-3}^{2}$ is the metric on the unit $S^{d-3}$. We assume that the two black holes have equal mass, $M_{+}=M_{-}=M$, in what follows.

We focus on particle dynamics in the dihole spacetime. Let $p_{\mu}$ be canonical momenta conjugate to coordinates, $x^{\mu}$. The Hamiltonian of a freely falling particle with unit/zero mass is given by

\footnotetext{
${ }^{3}$ This is a solution in the $d$-dimensional Einstein-Maxwell theory, whose action is given by

$$
S=\frac{1}{16 \pi G} \int \mathrm{d}^{d} x \sqrt{-g}\left(R-F_{\mu \nu} F^{\mu \nu}\right),
$$

where $R$ is the Ricci tensor, and $F_{\mu \nu}$ is the field strength of the gauge field, and we have restored the $d$-dimensional Newton constant $G$.
} 


$$
\begin{aligned}
H & =\frac{1}{2} g^{\mu \nu} p_{\mu} p_{\nu} \\
& =\frac{1}{2}\left[-U^{2} p_{t}^{2}+U^{-2 /(d-3)}\left(p_{z}^{2}+p_{\rho}^{2}+\frac{1}{\rho^{2}} \gamma^{a b} p_{a} p_{b}\right)\right],
\end{aligned}
$$

where $g^{\mu \nu}$ is the inverse metric of $g_{\mu \nu}$, and $\gamma^{a b}$ is the inverse of the metric on $S^{d-3}$. The momentum $p_{t}=-E$ is a conserved energy because $H$ is independent of time $t$. The quadratic quantity $\gamma^{a b} p_{a} p_{b}=L^{2}$ is also a constant of motion associated with spherical symmetry on $S^{d-3}$.

We consider stationary orbits on which a particle takes constant $z$ and $\rho$. The on-shell condition of geodesic motion $g^{\mu \nu} p_{\mu} p_{\nu}+\kappa=0$, where $\kappa$ is squared particle mass, yields

$$
\begin{gathered}
U^{2(4-d) /(d-3)}\left(\dot{z}^{2}+\dot{\rho}^{2}\right)+V=E^{2}, \\
V\left(\rho, z ; L^{2}\right)=\frac{L^{2}}{\rho^{2} U^{2(d-2) /(d-3)}}+\frac{\kappa}{U^{2}},
\end{gathered}
$$

where the dots denote the derivatives with respect to an affine parameter along the geodesic. We call $V$ the effective potential. Let us focus on particles with $\kappa=1$ staying in stationary orbits. The conditions of the stationary orbits for $V$ and $V_{i}:=\partial_{i} V(i=z, \rho)$ are written as

$$
\begin{gathered}
V_{z}=-\frac{2 U_{z}}{U^{3}}\left(\frac{d-2}{d-3} \frac{L^{2}}{\rho^{2} U^{2 /(d-3)}}+\kappa\right)=0, \\
V_{\rho}=-\frac{2 L^{2}}{\rho^{3} U^{2(d-2) /(d-3)}}-\frac{2 U_{\rho}}{U^{3}}\left(\frac{d-2}{d-3} \frac{L^{2}}{\rho^{2} U^{2 /(d-3)}}+\kappa\right)=0,
\end{gathered}
$$

$$
V=E^{2}
$$

respectively, where $U_{i}:=\partial_{i} U(i=z, \rho)$ take the forms

$$
\begin{aligned}
& U_{z}=-(d-3) M\left(\frac{z+a}{r_{+}^{d-1}}+\frac{z-a}{r_{-}^{d-1}}\right), \\
& U_{\rho}=-(d-3) M \rho\left(\frac{1}{r_{+}^{d-1}}+\frac{1}{r_{-}^{d-1}}\right),
\end{aligned}
$$

respectively. Solving the condition (11) for $L^{2}$, we have

$$
L^{2}=L_{0}^{2}:=-\frac{(d-3) \rho^{3} U_{\rho} U^{2 /(d-3)}}{(d-3) U+(d-2) \rho U_{\rho}} .
$$

Note that $L_{0}^{2}$ must not be negative, which is a necessary condition for the existence of a stationary orbit. From the condition (12) together with $L_{0}^{2}$, we obtain

$$
E^{2}=E_{0}^{2}:=V\left(\rho, z ; L_{0}^{2}\right)=\frac{(d-3) U+\rho U_{\rho}}{U^{2}\left[(d-3) U+(d-2) \rho U_{\rho}\right]},
$$

which also must not be negative. Note that if $L_{0}^{2} \geq 0$, we have $E_{0}^{2}>0$. The condition (10) implies $U_{z}=0$, which defines curves on the $\rho-z$ plane. These curves are distributed in the range $|z|<a$ and always include $z=0$. Now we define a family of the curves on the $\rho-z$ plane satisfying $L_{0}^{2} \geq 0$,

$$
\gamma_{0}:=\left\{(\rho, z) \mid U_{z}=0, L_{0}^{2} \geq 0\right\}
$$

which provides the sequence of stationary orbits. If evaluated at points on $\gamma_{0}$, the quantities $L_{0}$ and $E_{0}$ give the angular momentum and energy of a particle in stationary orbits, respectively. Note that all of the stationary orbits we are considering here are circular orbits. This is because, due to the spherical symmetry on $S^{d-3}$, particles in stationary orbits always move geodesically along a certain great circle on the sphere. Therefore, we will call the stationary orbit a circular orbit.

Now we look for a subset of $\gamma_{0}$ in which the stationary orbits are stable. Let $\left(V_{i j}\right)$ be the Hessian matrix of $V$, where $V_{i j}:=\partial_{j} \partial_{i} V$. We define $h$ and $k$ as the determinant and the trace of $\left(V_{i j}\right)$, i.e., $h\left(\rho, z ; L^{2}\right):=\operatorname{det}\left(V_{i j}\right)$ and $k\left(\rho, z ; L^{2}\right):=\operatorname{tr}\left(V_{i j}\right)$, respectively. In terms of $h$ and $k$, we define the region $D$ in which the circular orbits are stable as

$$
D:=\left\{(\rho, z) \mid h_{0}>0, k_{0}>0, L_{0}^{2} \geq 0\right\}
$$

where $h_{0}$ and $k_{0}$ are defined by

$$
h_{0}:=\left.h\left(\rho, z ; L_{0}^{2}\right)\right|_{U_{z}=0},
$$

$$
k_{0}:=\left.k\left(\rho, z ; L_{0}^{2}\right)\right|_{U_{z}=0},
$$

respectively. The restriction denoted by $U_{z}=0$ means that we have directly dropped the terms including $U_{z}$. Thus, we can visualize the sequence of stable circular orbits by $\gamma_{0}$ and $D$ in the $\rho-z$ plane. Thus, we can visualize the sequence of stable circular orbits by the overlap of $\gamma_{0}$ and $D$ in the $\rho-z$ plane. Note that $D$ only serves to find the subset of $\gamma_{0}$.

Here, we summarize the quantities $E_{0}, L_{0}, h_{0}$, and $k_{0}$ evaluated on $z=0$. We introduce the $(d-2)$-dimensional radial coordinate defined by $R:=\sqrt{\rho^{2}+a^{2}}$ for simplification of both calculations and expressions, where note that $R \geq a$. Let us use units in which $M=1$ in what follows. The energy and angular momentum of a particle in a circular orbit on $z=0$ are given by 


$$
\begin{gathered}
E_{0}^{2}(\rho, 0)=\frac{R^{2(d-3)}\left(R^{d-1}+2 a^{2}\right)}{\left(R^{d-3}+2\right)^{2} f}, \\
L_{0}^{2}(\rho, 0)=\frac{2(d-3)\left(R^{2}-a^{2}\right)^{2}\left(R^{d-3}+2\right)^{2 /(d-3)}}{R^{2} f},
\end{gathered}
$$

respectively, where

$$
f(R):=R^{d-1}-2(d-3) R^{2}+2(d-2) a^{2} .
$$

Note that $f(R)$ must be always positive on $\gamma_{0}$. The derivatives of $E_{0}(\rho, 0)$ and $L_{0}(\rho, 0)$ with respect to $R$ are given by

$$
\begin{aligned}
\frac{\mathrm{d} E_{0}(\rho, 0)}{\mathrm{d} R} & =\frac{(d-3) g}{R\left(R^{d-3}+2\right)\left(R^{d-1}+2 a^{2}\right) f}, \\
\frac{\mathrm{d} L_{0}(\rho, 0)}{\mathrm{d} R} & =\frac{g}{2 R\left(R^{d-3}+2\right)\left(R^{2}-a^{2}\right) f},
\end{aligned}
$$

respectively, where

$$
\begin{aligned}
g(R):= & 8(d-2) a^{4}+\left[2(3 d-1) R^{d-1}\right. \\
& \left.+(d-1) R^{2(d-2)}-8(d-4) R^{2}\right] a^{2} \\
& -6(d-3) R^{d+1}-(d-5) R^{2(d-1)} .
\end{aligned}
$$

The signs of these derivatives on $\gamma_{0}$ are determined by that of $g(R)$. The quantities $h_{0}$ and $k_{0}$ evaluated on $z=0$ are given by

$$
\begin{aligned}
& \quad h_{0}(\rho, 0)=\frac{16(d-3)^{2} R^{2(2 d-9)}\left[R^{2}-(d-1) a^{2}\right] g}{\left(R^{d-3}+2\right)^{6} f^{2}}, \quad \\
& k_{0}(\rho, 0) \\
& =\frac{4(d-3) R^{2(d-5)}\left[g+R^{2}\left(R^{d-3}+2\right)^{2}\left[R^{2}-(d-1) a^{2}\right]\right]}{\left(R^{d-3}+2\right)^{4} f},
\end{aligned}
$$

respectively.

Now let us discuss some $d$-independent properties. One property common to these systems is that the center of the system (i.e., the center of the two black holes) is located outside the horizon. This fact leads to a common property in the structure of $V$. On $z=0$, the expansion of $V$ around $\rho=0$ is written as

$$
V(\rho, 0)=\frac{a^{2(d-2)} L^{2}}{\left(a^{d-3}+2\right)^{2(d-2) /(d-3)} \rho^{2}}+O\left(\rho^{0}\right) .
$$

Note that the power of $\rho$ in the leading term does not depend on $d$. If $L \neq 0$, then $V(\rho, 0)$ diverges in the limit $\rho \rightarrow 0$, which shows the appearance of the centrifugal barrier near the center. Since gravitational force acts attractively, there always exists a stable balance between the gravitational force and the centrifugal force in the $\rho$ direction near the center. In the $z$ direction, $V$ makes a local maximum in the range $a \leq R<a \sqrt{d-1}$ because $V_{z}(\rho, 0)=0$ from the reflection symmetry, and $V_{z z}(\rho, 0)>0$, where

$$
\begin{aligned}
V_{z z}(\rho, 0)= & \frac{4\left[R^{2}-(d-1) a^{2}\right]}{\left(R^{2}-a^{2}\right)\left(R^{d-3}+2\right)^{3}}\left[\frac{(d-2) L^{2} R^{2(d-4)}}{\left(R^{d-3}+2\right)^{2 /(d-3)}}\right. \\
& \left.+(d-3) R^{2(d-5)}\left(R^{2}-a^{2}\right)\right] .
\end{aligned}
$$

Hence, $V$ always makes a saddle point near the center, so that no stable circular orbits appear there.

\section{STABLE/UNSTABLE CIRCULAR ORBITS}

$$
\text { A. } d=5
$$

We consider how the sequence of circular orbits varies as the dihole separation gradually decreases from a sufficiently large value in the 5D MP spacetime. We first check the explicit forms of quantities evaluated on the symmetric plane $z=0$. From Eqs. (21) and (22), $E_{0}^{2}$ and $L_{0}^{2}$ in $d=5$ are given by

$$
\begin{gathered}
E_{0}^{2}(\rho, 0)=\frac{R^{4}\left(R^{4}+2 a^{2}\right)}{\left(R^{2}+2\right)^{2} f}, \\
L_{0}^{2}(\rho, 0)=\frac{4\left(R^{2}-a^{2}\right)^{2}\left(R^{2}+2\right)}{R^{2} f},
\end{gathered}
$$

respectively, where

$$
f(R)=R^{4}-4 R^{2}+6 a^{2} .
$$

From Eqs. (24) and (25), the derivatives of $E_{0}(\rho, 0)$ and $L_{0}(\rho, 0)$ take the forms

$$
\begin{aligned}
& \frac{\mathrm{d} E_{0}(\rho, 0)}{\mathrm{d} R}=\frac{2 g}{R\left(R^{2}+2\right)\left(R^{4}+2 a^{2}\right) f}, \\
& \frac{\mathrm{d} L_{0}(\rho, 0)}{\mathrm{d} R}=\frac{g}{2 R\left(R^{2}+2\right)\left(R^{2}-a^{2}\right) f},
\end{aligned}
$$

respectively, where

$$
\begin{aligned}
g(R)= & 4\left[\left(a^{2}-3\right) R^{6}+R^{2}\left(R^{2}-4 a^{2}\right)\right. \\
& \left.+\left(7 a^{2}-1\right) R^{4}+2 a^{2}\left(R^{2}+3 a^{2}\right)\right] .
\end{aligned}
$$

From Eqs. (27) and (28), the quantities $h_{0}$ and $k_{0}$ take the forms 


$$
\begin{gathered}
h_{0}(\rho, 0)=\frac{64 R^{2}\left(R^{2}-4 a^{2}\right) g}{\left(R^{2}+2\right)^{6} f^{2}}, \\
k_{0}(\rho, 0)=\frac{8\left[g+R^{2}\left(R^{2}+2\right)^{2}\left(R^{2}-4 a^{2}\right)\right]}{\left(R^{2}+2\right)^{4} f},
\end{gathered}
$$

respectively. Besides $z=0$, there is the following branch of $U_{z}=0$ :

$$
z=z_{0}(R):= \pm \sqrt{R(2 a-R)},
$$

where $a<R \leq 2 a$ (i.e., $0<\rho \leq \sqrt{3} a$ ). The particle energy and angular momentum in a circular orbit on $z=z_{0}$ are given by

$$
\begin{gathered}
E_{0}^{2}\left(\rho, z_{0}\right)=\frac{4 a^{2}(R-a)^{3}(4 a R+1)}{[2 a(R-a)+1]^{2} F}, \\
L_{0}^{2}\left(\rho, z_{0}\right)=\frac{[2 a(R-a)+1](R+a)^{2}}{a F},
\end{gathered}
$$

respectively, where

$$
F(R):=4 a R^{2}-\left(4 a^{2}+1\right) R-3 a .
$$

Note that $F(R)$ must be always positive on $\gamma_{0}$. The derivatives of $E_{0}\left(\rho, z_{0}\right)$ and $L_{0}\left(\rho, z_{0}\right)$ with respect to $R$ are given by

$$
\frac{\mathrm{d} E_{0}\left(\rho, z_{0}\right)}{\mathrm{d} R}=\frac{G}{(R-a)[2 a(R-a)+1](4 a R+1) F},
$$

$$
\frac{\mathrm{d} L_{0}\left(\rho, z_{0}\right)}{\mathrm{d} R}=\frac{G}{2(R+a)[2 a(R-a)+1] F},
$$

respectively, where

$$
G(R):=8 a^{2}(R-a)^{3}-4 a R^{2}-\left(28 a^{2}+1\right) R+a\left(8 a^{2}-5\right) .
$$

The signs of these quantities on $\gamma_{0}$ are determined by that of $G(R)$. The quantities $h_{0}$ and $k_{0}$ take the forms

$$
h_{0}\left(\rho, z_{0}\right)=\frac{128 a^{2}(R-a)^{3}(2 a-R) G}{R F^{2}[2 a(R-a)+1]^{6}},
$$

$$
k_{0}\left(\rho, z_{0}\right)=\frac{8 a^{2}(R-a)\left[8 a^{2} R^{4}-4 a\left(4 a^{2}+1\right) R^{3}+\left(8 a^{4}-8 a^{2}-1\right) R^{2}+12 a^{3} R+3 a^{2}\right]}{R^{2}[2 a(R-a)+1]^{4} F},
$$

respectively. The sign of $h_{0}\left(\rho, z_{0}\right)$ on $\gamma_{0}$ is also determined by that of $G(R)$.

For $a \gg 1$, the sequence of circular orbits is of a typical form as shown in Fig. 1(a). The black solid line shows $\gamma_{0}$, and the blue shaded region shows the region $D$. On $z=0$, stable circular orbits exist within the range of $\sqrt{3} a \leq \rho<\infty$ because both $h_{0}(\rho, 0)$ and $k_{0}(\rho, 0)$ are positive in this range [i.e., $g(R) \geq 0$ ]. The green point $(\rho, z)=(\sqrt{3} a, 0)$ corresponds to a marginally stable circular orbit (MSCO), where $h_{0}$ vanishes, i.e., $g(R)=0$. Furthermore, stable circular orbits also appear on the branch $z=z_{0}$ and extend from the MSCO to the ISCOs, which correspond to the red points, where $h_{0}$ also vanishes, i.e., $G(R)=0$. On the sequences of stable circular orbits, we have $\mathrm{d} E_{0} / \mathrm{d} R>0$ and $\mathrm{d} L_{0} / \mathrm{d} R>0$. The sequences $\gamma_{0}$ appearing outside the region $D$ are those of unstable circular orbits. They are distributed in $0 \leq \rho \leq \sqrt{3} a$ on the $z=0$ plane and in $R_{\mathrm{L}}<R \leq 2 a$ on $z=z_{0}$, where $R_{\mathrm{L}}$ is given as a solution to $F=0$,

$$
R_{\mathrm{L}}:=\frac{1}{8 a}\left[4 a^{2}+1+\sqrt{16 a^{4}+56 a^{2}+1}\right] .
$$

The positions $(R, z)=\left(R_{\mathrm{L}}, z_{0}\left(R_{\mathrm{L}}\right)\right)$ are shown by white points in Fig. 1(a). Note that $E_{0}^{2}$ and $L_{0}^{2}$ become infinite here. This corresponds to photon circular orbits because the divergence of the unit mass quantities indicates the massless limit and the ratio $L_{0} / E_{0}$ remains finite even in this limit. Consequently, we find that the last circular orbits correspond to unstable photon circular orbits.

As the value of $a$ gradually decreases, the ISCOs approach the MSCO. Eventually, they merge when $a$ reaches a specific value, $a_{0}$, as shown in Fig. 1(b). Then the two real roots of $h_{0}\left(\rho, z_{0}\right)=0$ must be degenerate at $R=2 a$, i.e.,

$$
G(2 a)=a\left(8 a^{4}-64 a^{2}-7\right)=0 .
$$

As a solution to this equation, we define

$$
a_{0}:=\frac{1}{2} \sqrt{16+3 \sqrt{30}}=2.8474 \cdots .
$$

When $a$ is within $a_{0} \geq a \geq a_{*}$, where

$$
a_{*}:=\sqrt{3},
$$




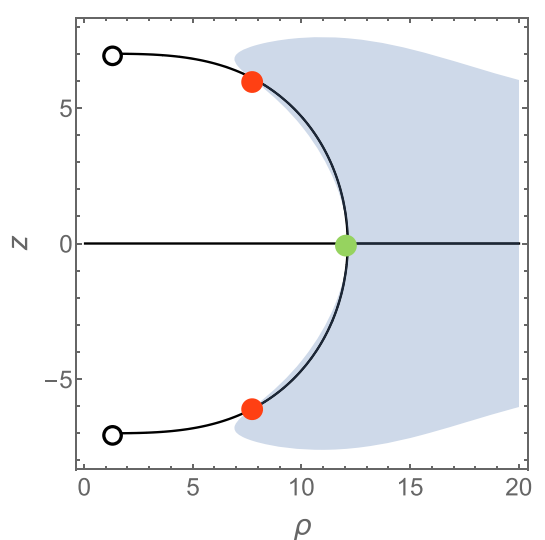

(a) $a=7$

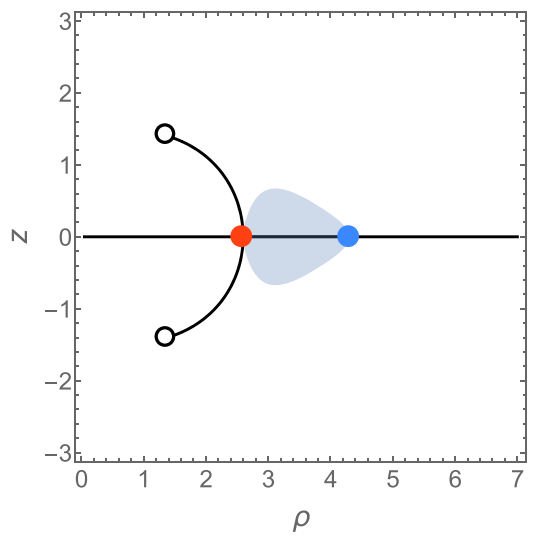

(d) $a=1.5$

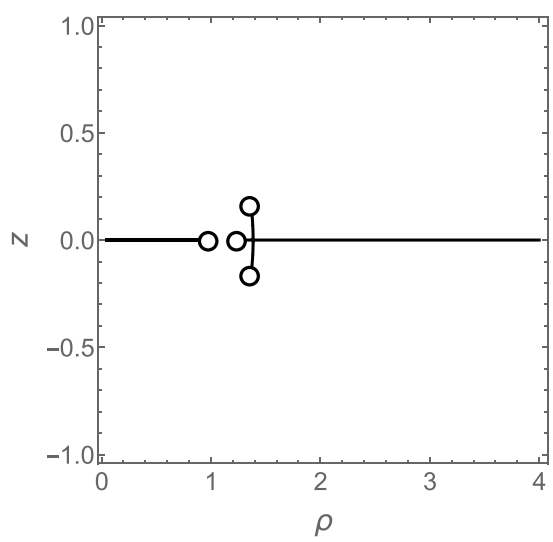

(g) $a=0.8$

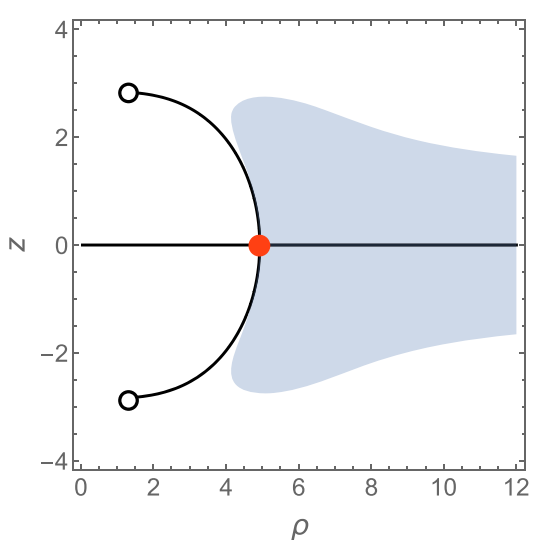

(b) $a=a_{0}=2.8474 \cdots$

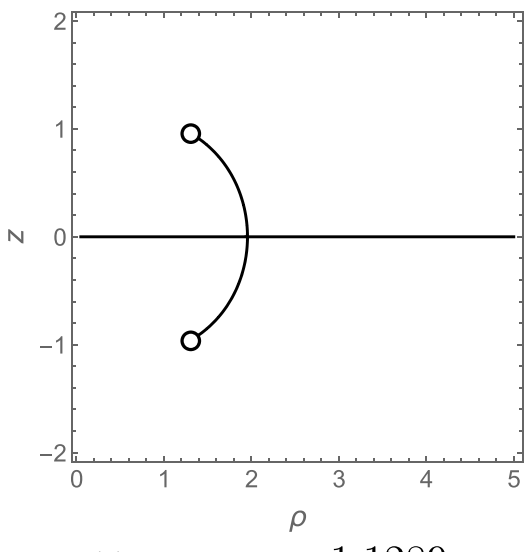

(e) $a=a_{\mathrm{c}}=1.1289 \ldots$

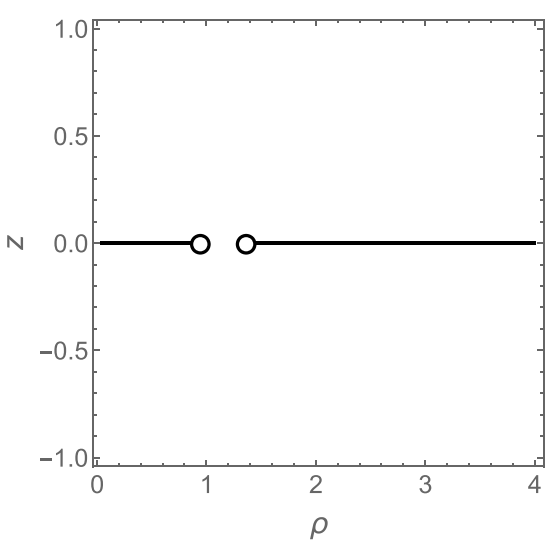

(h) $a=a_{1}=0.7905 \cdots$

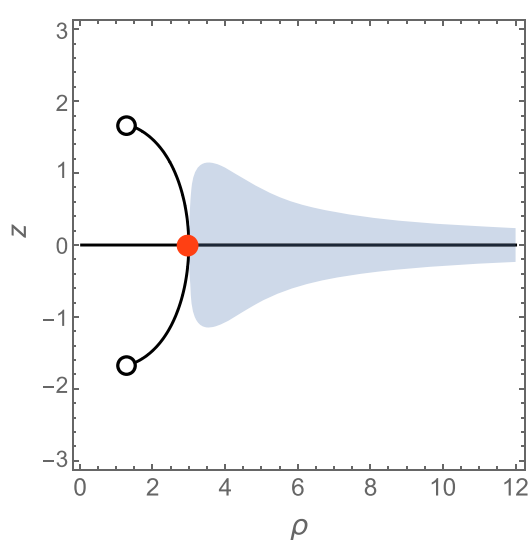

(c) $a=a_{*}=\sqrt{3}$

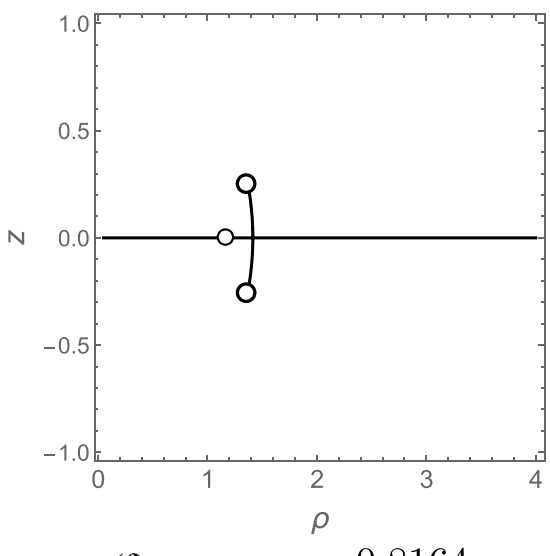

(f) $a=a_{\infty}=0.8164 \cdots$

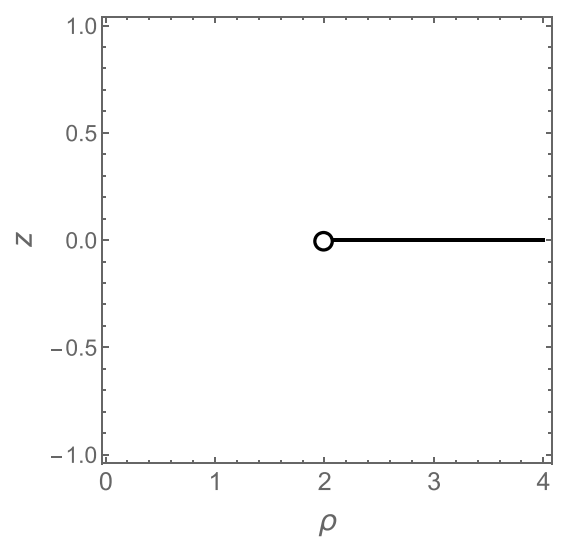

(i) $a=0$

FIG. 1. Sequences of circular orbits in the 5D MP dihole spacetimes. Units are in which $M=1$ are used. Each black solid line shows $\gamma_{0}$, which is a sequence of circular orbits. Each blue shaded region shows $D$, in which circular orbits are stable. The boundaries of $D$ are determined by $h_{0}=0$. Points colored by red, green, and blue denote the ISCO, MSCO, and OSCO, respectively. Each white point indicates an unstable photon circular orbit. (a) $\gamma_{0}$ between the red and green points overlaps $D$. (b) $\gamma_{0}$ between the red and white points does not overlap $D$. 
the quantities $h_{0}(\rho, 0)$ and $k_{0}(\rho, 0)$ are not negative in a half-line region $\sqrt{3} a \leq \rho<\infty,{ }^{4}$ and hence, stable circular orbits appear there. The point $(\rho, z)=(\sqrt{3} a, 0)$ corresponds to the ISCO, colored by red in Figs. 1(b) and 1(c), where $h_{0}(\rho, 0)=0$.

Let us now consider the reason why stable circular orbits exist at infinity for $a \geq a_{*}$. The asymptotic expansion of $V(\rho, 0)$ at $\rho \rightarrow \infty$ is given by

$V(\rho, 0)-1=-\frac{4-L^{2}}{\rho^{2}}+\frac{4\left(a^{2}-3\right)+6\left(4-L^{2}\right)}{\rho^{4}}+O\left(\rho^{-6}\right)$.

If $L^{2}<4$, the leading term, the sum of the Newtonian gravitational potential and the centrifugal potential, is negative, and if $4\left(a^{2}-3\right)+6\left(4-L^{2}\right)>0$, the subleading term is positive. Furthermore, if $0<4-L^{2} \ll 1$, then we find a local minimum point of $V(\rho, 0)$ in the asymptotic region,

$$
\rho \simeq 2 \sqrt{\frac{2\left(a^{2}-3\right)}{4-L^{2}}} .
$$

In order for this value to be a nonzero real number, we have $a>a_{*}$. For the marginal case $a=a_{*}$, we also find a local minimum point of $V(\rho, 0)$ in the asymptotic region, ${ }^{5}$ and therefore, stable circular orbits appear in $3 \leq \rho<\infty$ [see Fig. 1(c)]. This is why we can conclude that there exist stable circular orbits even at infinity in the range $a \geq a_{*}$. Note that in the case of a 5D static and spherically symmetric black hole, there is no stable circular orbit $[3,4]$. This implies that the existence of stable circular orbits is due to the dihole separation, and furthermore, in the range of $a \geq a_{*}$, its effects can be observed at infinity.

${ }^{4}$ If $a \geq \sqrt{3}$, then $g(R)>0$ and $f(R)>0$ in the range $2 a \leq R<\infty$.

${ }^{5}$ The expansion (52) around $a=a_{*}$ is

$$
\begin{aligned}
V(\rho, 0)-1 & =\sum_{l=1}^{\infty} V_{(2 l)}, \quad V_{(2)}=-\epsilon / \rho^{2}, \\
V_{(4)} & =6 \epsilon / \rho^{4}, \quad V_{(6)}=14(2-3 \epsilon) / \rho^{6},
\end{aligned}
$$

where $\epsilon=4-L^{2}>0$. Comparing each term in the limit $\rho \rightarrow \infty$ and $\epsilon \rightarrow 0$, we have

$$
\begin{aligned}
& \left|V_{(4)} / V_{(2)}\right|=O\left(\rho^{-2}\right), \quad\left|V_{(6)} / V_{(2)}\right|=O\left(\epsilon^{-1} \rho^{-4}\right), \\
& \left|V_{(6)} / V_{(4)}\right|=O\left(\epsilon^{-1} \rho^{-2}\right) .
\end{aligned}
$$

If $\epsilon$ and $\rho$ satisfy $\epsilon \rho^{4}=O(1)$ in this limit, $V_{(2)}$ and $V_{(6)}$ are dominant even in the asymptotic region, and $V_{(4)}$ is negligible. Then, we find a local minimum point of $V(\rho, 0)$ in the asymptotic region, $\rho \simeq \sqrt[4]{84} \epsilon^{-1 / 4}$.
When $a<a_{*}$, there is no longer stable circular orbit at infinity. However, for $a_{*}>a>a_{\mathrm{c}}$, where $a_{\mathrm{c}}$ is determined by the discussion below, a sequence of stable circular orbits draws a segment with finite length on $z=0$, as shown in Fig. 1(d). The segment appears in the interval

$$
R_{\mathrm{ISCO}} \leq R \leq R_{\mathrm{OSCO}}
$$

where $R_{\mathrm{ISCO}}:=2 a$ and $R_{\mathrm{OSCO}}$ correspond to, respectively, the radii of the ISCO and the outermost stable circular orbit (OSCO), which solve $h_{0}(\rho, 0)=0$ and are denoted, respectively, by the red point and the blue point in Fig. 1(d). Both radii of the ISCO and the OSCO monotonically decrease as $a$ decreases and are degenerate at $a=a_{\mathrm{c}}$, where

$$
a_{\mathrm{c}}:=\frac{\sqrt{10+6 \sqrt{3}}}{4}=1.1289 \cdots,
$$

which are determined by the degenerate condition of two roots of $h_{0}(\rho, 0)=0$ at $R=2 a$, i.e.,

$$
g(2 a)=8 a^{4}\left(32 a^{4}-40 a^{2}-1\right)=0 .
$$

The sequences of unstable circular orbits are distributed to $0 \leq R \leq 2 a$ and $R_{\mathrm{OSCO}}<R<\infty$ on $z=0$ and to $R_{\mathrm{L}}<R \leq 2 a$ on $z=z_{0}$.

In the range $a \leq a_{\mathrm{c}}$, there is no overlapping set of $\gamma_{0}$ and $D$, i.e., there is no stable circular orbit. Therefore, we focus only on the $a$-dependent deformation of $\gamma_{0}$. As $a$ decreases from $a_{\mathrm{c}}$ to $a_{\infty}$, the outline of $\gamma_{0}$ remains the same as that in Fig. 1(e), where

$$
a_{\infty}:=\frac{\sqrt{6}}{3}=0.8164 \cdots
$$

When $a=a_{\infty}$, the function $f(R)$ in Eq. (33) vanishes only at $R=\sqrt{2}$ (i.e., $\rho=2 \sqrt{3} / 3$ ), where $E_{0}$ and $L_{0}$ diverge. This means that there exists an unstable circular orbit not for massive particles but for massless particles, which are shown by a white point on the $z=0$ plane in Fig. 1(f).

In the range $a<a_{\infty}$, the sequence of $\gamma_{0}$ on $z=0$ separates into two parts. Note that the outer boundary of the inner sequence and the inner boundary of the outer sequence are unstable photon circular orbits, which are located at $R=\left[2 \pm \sqrt{2\left(2-3 a^{2}\right)}\right]^{1 / 2}$. When $a=a_{1}$, the sequence of $\gamma_{0}$ on $z=z_{0}$ vanishes at $(R, z)=(2 a, 0)$, and at the same time the inner boundary of the outer sequence on the $z=0$ plane vanishes at the same point, i.e., $R_{\mathrm{L}}=2 a=\left[2+\sqrt{2\left(2-3 a^{2}\right)}\right]^{1 / 2}$, where

$$
a_{1}:=\frac{\sqrt{10}}{4}=0.7905 \cdots
$$


In $a<a_{1}$, the sequence on $z=z_{0}$ no longer appears, and the inner and outer sequences on $z=0$ only appear. In the limit $a \rightarrow 0$, the geometry approaches the extremal Reissner-Nordström black hole spacetime with mass 2 . Then the inner sequence on $z=0$ disappears, and the inner boundary of the outer sequence, the unstable photon circular orbit, limits to $(\rho, z)=(2,0)$ (see the Appendix).

We summarize the dependence of characteristic radii on $a$ in Fig. 2.

\section{B. $d \geq 6$}

We consider the dependence of sequences of circular orbits on the separation in the 6D MP dihole spacetime. We summarize several quantities associated with circular orbits and their stability. From Eqs. (21) and (22), $E_{0}^{2}$ and $L_{0}^{2}$ in $d=6$ are given by

$$
\begin{gathered}
E_{0}^{2}(\rho, 0)=\frac{R^{6}\left(R^{5}+2 a^{2}\right)}{\left(R^{3}+2\right)^{2} f}, \\
L_{0}^{2}(\rho, 0)=\frac{6\left(R^{2}-a^{2}\right)^{2}\left(R^{3}+2\right)^{2 / 3}}{R^{2} f},
\end{gathered}
$$

respectively, where

$$
f(R):=R^{5}-6 R^{2}+8 a^{2} .
$$

From Eqs. (24) and (25), the derivatives of $E_{0}(\rho, 0)$ and $L_{0}(\rho, 0)$ take the form

$$
\begin{aligned}
& \frac{\mathrm{d} E_{0}(\rho, 0)}{\mathrm{d} R}=\frac{3 g}{R\left(R^{3}+2\right)\left(R^{5}+2 a^{2}\right) f}, \\
& \frac{\mathrm{d} L_{0}(\rho, 0)}{\mathrm{d} R}=\frac{g}{2 R\left(R^{3}+2\right)\left(R^{2}-a^{2}\right) f},
\end{aligned}
$$

respectively, where

$$
\begin{aligned}
g(R):= & -2\left(R^{2}-a^{2}\right)\left[5 R^{2}\left(R^{3}+2\right)+2 f\right] \\
& -R^{2}\left(R^{2}-5 a^{2}\right)\left(R^{3}+2\right)^{2} .
\end{aligned}
$$

From Eqs. (27) and (28), the quantities $h_{0}$ and $k_{0}$ take the forms

$$
\begin{gathered}
h_{0}(\rho, 0)=\frac{144 R^{6}\left(R^{2}-5 a^{2}\right) g}{\left(R^{3}+2\right)^{6} f^{2}}, \\
k_{0}(\rho, 0)=-\frac{24 R^{2}\left(R^{2}-a^{2}\right)\left[5 R^{2}\left(R^{3}+2\right)+2 f\right]}{\left(R^{3}+2\right)^{4} f},
\end{gathered}
$$

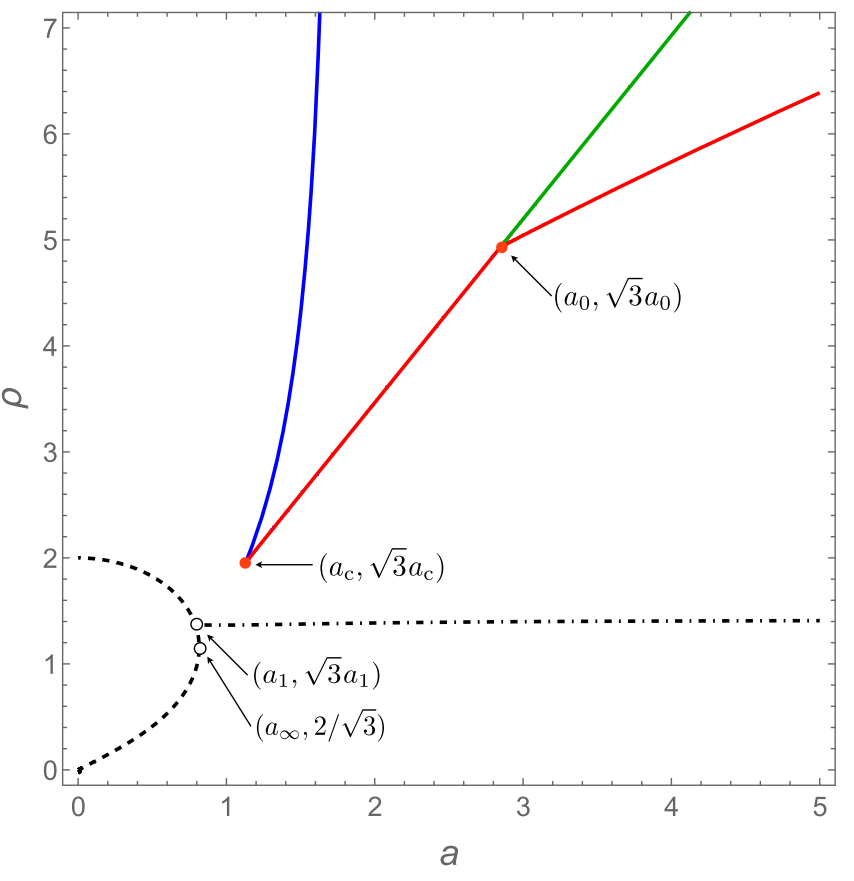

FIG. 2. Dependence of characteristic radii on $a$ in the 5D MP dihole spacetime. Units are in which $M=1$ are used. The curves colored by green, red, and blue show the MSCO, ISCO, and OSCO, respectively. Note that the OSCO appears only in the range $a_{\mathrm{c}} \leq a<a_{*}$. Dashed and dot-dashed curves colored by black show unstable photon circular orbits on $z=0$ and $z=z_{0}$, respectively.

respectively. Besides $z=0$, there exists the following branch that satisfies $U_{z}=0$ :

$$
z=z_{0}(R)
$$

Unlike Eq. (39) in the case $d=5$, however, it is not possible to write $z_{0}$ explicitly in this case because the condition is given as an algebraic equation of the fifth degree or more.

In the case $a \gg 1$, the sequences of circular orbits typically show the shape depicted in Fig. 3(a), where black solid curves are $\gamma_{0}$. The set $\gamma_{0}$ contains $z=0$ and a part of $z=z_{0}$. The angular momentum $L_{0}^{2}$ and energy $E_{0}^{2}$ diverge at the boundaries of $\gamma_{0}$ on $z=z_{0}$ corresponding to the two white points, where there exist unstable circular orbits for massless particles rather than massive particles. As is seen from the asymptotic expansion of $V(\rho, 0)$ at $\rho \rightarrow \infty$,

$$
V(\rho, 0)-1=\frac{L^{2}}{\rho^{2}}-\frac{4}{\rho^{3}}+O\left(\rho^{-5}\right)
$$

the leading term "the centrifugal potential" and the subleading term "the Newtonian gravitational potential" do not make a potential well, so that there is no stable circular orbit in the asymptotic region. The nonexistence of stable 


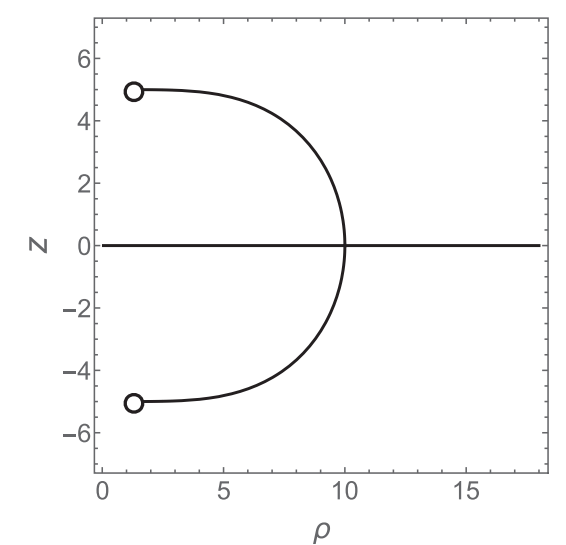

(a) $a=5$

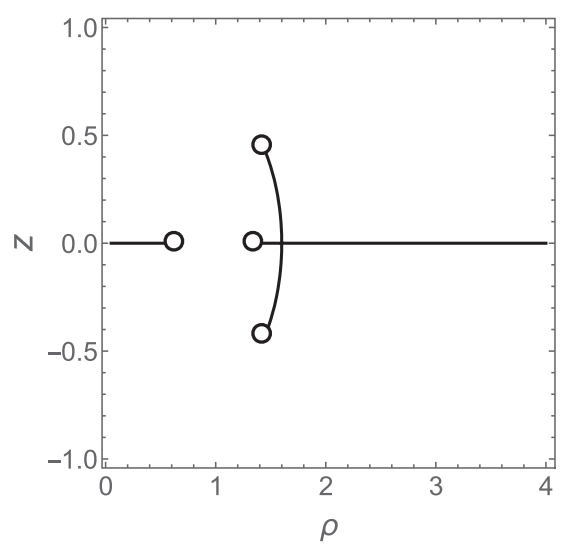

(c) $a=0.8$

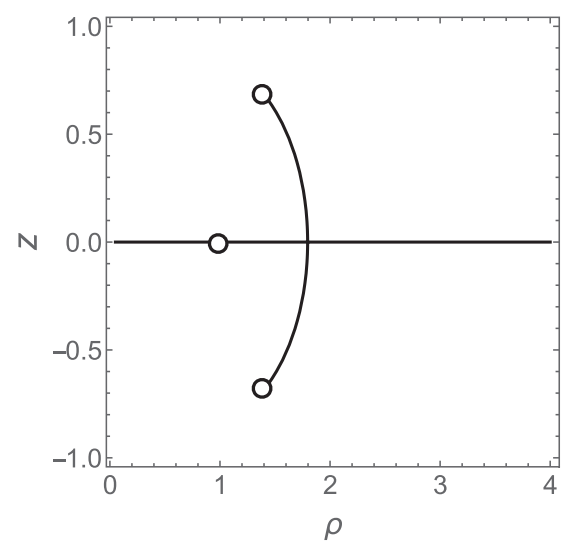

(b) $a=a_{\infty}=0.8981 \cdots$

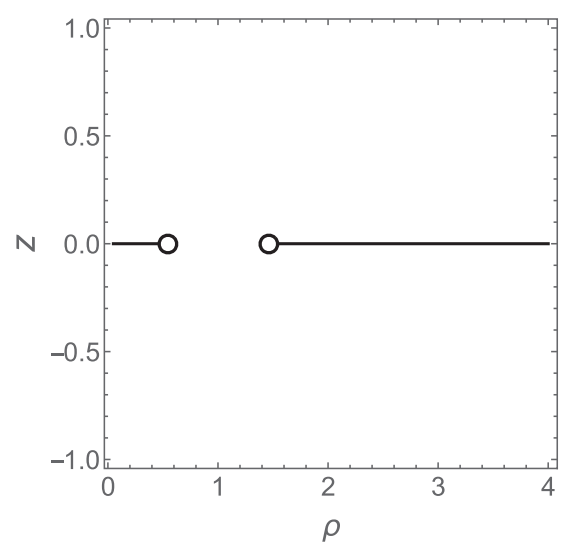

(d) $a=a_{1}=0.7328 \cdots$

FIG. 3. Sequences of circular orbits in the 6D MP dihole spacetimes. Units in which $M=1$ are used. Each black solid line shows $\gamma_{0}$. Each white point indicates an unstable photon circular orbit.

circular orbits is not only in the asymptotic region but in the whole region. In fact, unlike in $d=4,5$, the region $D$ does not appear in $d=6$. We can interpret that the centrifugal force barrier at the center is ineffective to make a local minimum of $V$.

When $a$ takes the value

$$
a_{\infty}:=\frac{3}{10} \sqrt[6]{720}=0.8981 \cdots,
$$

there appears a (white) point on $z=0$ [corresponding to $R=R_{\infty}:=\sqrt[3]{12 / 5}$ (i.e., $\rho=\rho_{\infty}:=\sqrt[6]{720} \sqrt{11} / 10$ ] such that $f(R)$ vanishes, i.e., $E_{0}^{2}$ and $L_{0}^{2}$ diverge. This indicates the appearance of an unstable photon circular orbit.

As $a$ is further decreasing, the outer boundary of the inner sequence approaches the $z$ axis, whereas the inner boundary of the outer sequence goes away from the $z$ axis [see Fig. 3(c)], and at $a=a_{1}$, where

$$
a_{1}:=\frac{\sqrt[3]{22} \sqrt[6]{5}}{5}=0.7328 \cdots
$$

it coincides with the point $(\rho, z)=\left(2 a_{1}, 0\right)$, where $\rho_{1}=2 a_{1}$. At the same time, the boundaries of $\gamma_{0}$ on $z=z_{0}$ also limits to the same point. In other words, the three unstable photon circular orbits are degenerate there [see Fig. 3(d)].

For $a \geq a_{1}, \gamma_{0}$ contains only two sequences on $z=0$. In the limit $a \rightarrow 0$, the inner sequence vanishes at the origin, and the inner boundary of the outer sequence limits to $(\rho, z)=(2,0)$ (see the Appendix).

Finally we comment on how the $a$ dependence of the sequences of circular orbits changes as $d$ increases. At least for $d=7,8,9,10$, we have checked that the change in the sequences of circular orbits is qualitatively the same as in the case of $d=6$. Indeed, it can be seen from Figs. 4 that the dependence of the radii of unstable photon circular orbits on $a$ is qualitatively the same for $d=6,7, \ldots, 10$. The critical values of the separation and the radius, $a_{\infty}, \rho_{\infty}$, 


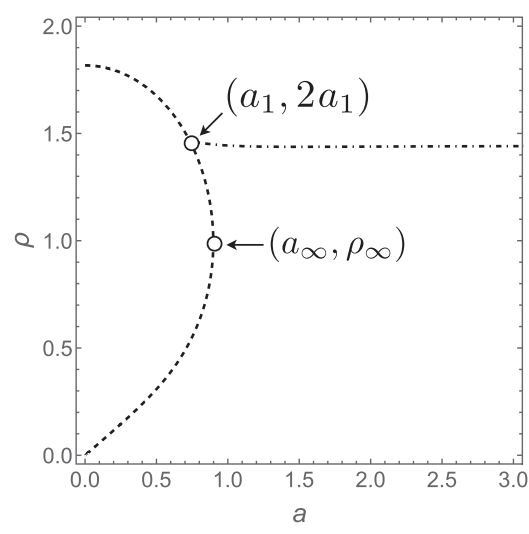

(a) $d=6$

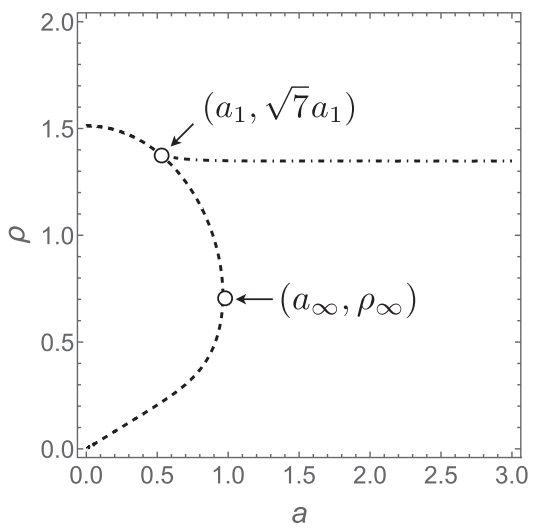

(d) $d=9$

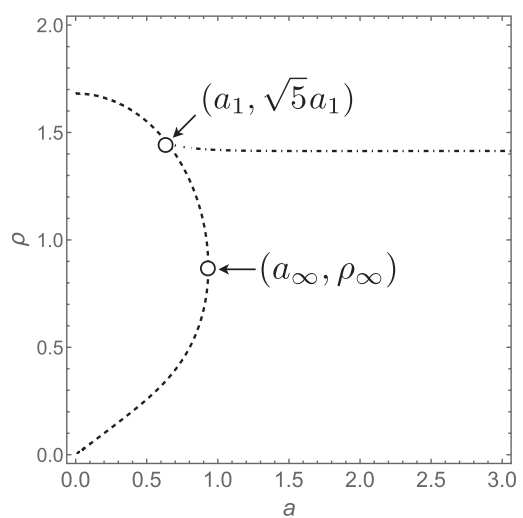

(b) $d=7$

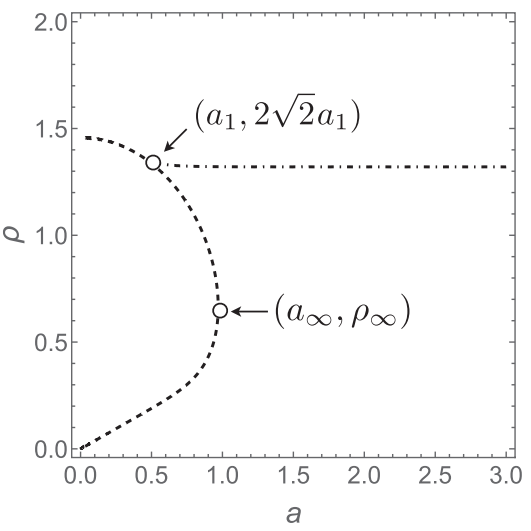

(e) $d=10$

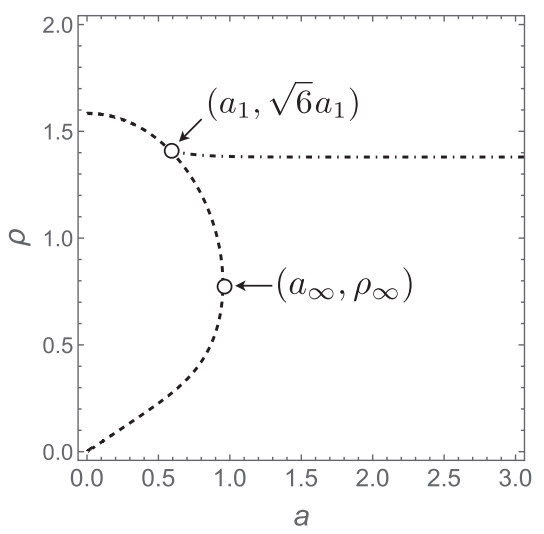

(c) $d=8$

FIG. 4. Dependence of characteristic radii on $a$ in $d=6,7, \ldots, 10$. Units in which $M=1$ are used. Dashed and dot-dashed curves colored by black show unstable photon circular orbits on $z=0$ and $z=z_{0}$, respectively.

$a_{1}$, and $\rho_{1}$, which are defined in the same sense as the $d=6$ case, are summarized in Table I. Note that $\rho_{1}=\sqrt{d-2} a_{1}$. This result suggests that the qualitative properties of stable/ unstable circular orbits are common in the MP dihole spacetime in $d \geq 6$.

\section{SUMMARY AND DISCUSSIONS}

We have considered the dynamics of particles, focusing on circular orbits, in the $d$-dimensional MP dihole spacetime $(d \geq 5)$. Using the on-shell conditions for geodesic motion, we have clarified the conditions for the existence of circular orbits in terms of a 2D effective potential and have also provided a prescription for determining whether these orbits are stable. Applying this formalism to the case of $d=5$, we have shown the dependence of sequences of stable/unstable circular orbits on the dihole separation. One of the most remarkable features is the appearance of stable circular orbits because it was shown in the previous works that they were not found

TABLE I. Critical values of $a_{1}, \rho_{1}, a_{\infty}$, and $\rho_{\infty}$ for $d=6,7,8,9,10$.

\begin{tabular}{|c|c|c|c|c|}
\hline$d$ & $a_{1}$ & $\rho_{1}$ & $a_{\infty}$ & $\rho_{\infty}$ \\
\hline 6 & $\frac{\sqrt[3]{22} \sqrt[6]{5}}{5}=0.7328 \cdots$ & $2 a_{1}=1.4656 \cdots$ & $\frac{3}{10} \sqrt[6]{720}=0.8981 \cdots$ & $\frac{\sqrt[6]{720} \sqrt{11}}{10}=0.9929 \ldots$ \\
\hline 7 & $\frac{\sqrt{2} \sqrt[4]{57}}{6}=0.6476 \cdots$ & $\sqrt{5} a_{1}=1.4481 \ldots$ & $\frac{4 \sqrt[4]{150}}{15}=0.9332 \ldots$ & $\frac{\sqrt{35} \sqrt[4]{24}}{15}=0.8729 \ldots$ \\
\hline 8 & $\frac{\sqrt[5]{58}}{\sqrt[10]{7^{7}}}=0.5769 \cdots$ & $\sqrt{6} a_{1}=1.4131 \cdots$ & $\frac{\sqrt[5]{3} \sqrt[5]{5} \sqrt[10]{7^{3}}}{21 \sqrt[10]{2}}=0.9517 \cdots$ & $\frac{\sqrt[5]{5} \sqrt[10]{7^{3}} \sqrt{51}}{\sqrt[10]{221}}=0.7848 \cdots$ \\
\hline 9 & $\frac{\sqrt[6]{82}}{4}=0.5210 \cdots$ & $\sqrt{7} a_{1}=1.3786 \cdots$ & $\frac{3 \sqrt[6]{3}}{\sqrt{14}}=0.9628$ & $\sqrt{\frac{5}{14}} 3^{1 / 6}=0.7176$. \\
\hline 10 & $\frac{1}{3} \sqrt[7]{\frac{110}{9}}=0.4766 \cdots$ & $2 \sqrt{2} a_{1}=1.3481 \cdots$ & $\frac{7 \sqrt[3]{7}}{6 \sqrt[14]{8} \sqrt[3]{9}}=0.9701 \cdots$ & $\frac{\sqrt[7]{7} \sqrt{23}}{6 \sqrt[14]{8} \sqrt[7]{9}}=0.6646$ \\
\hline
\end{tabular}


in single black holes with a spherical horizon $[3,4]$. Particularly for the large separation $a \geq a_{*}=\sqrt{3}$, they appear from the ISCO to infinity, whereas for $a_{\mathrm{c}}(=1.1289 \cdots)<a<a_{*}$, they exist only in a restricted region between the ISCO and the OSCO. Therefore, we can interpret this phenomenon as a result caused by the existence of two horizons. In other words, the center of the system is shifted off the horizon, and as a result, the effect of the centrifugal barrier near the center affects the existence of stable circular orbits in the intermediate region. Furthermore, the existence of the stable circular orbits in the asymptotic region is also affected by the power law of gravitational force specific to 5D. Let us note that as shown in Ref. [33,37] for the 4D MP dihole spacetime, stable circular orbits exist in the asymptotic region for arbitrary separations. Therefore, we can conclude that the appearance of the OSCO is a proper phenomenon of 5D.

In the cases of $d=6,7, \ldots, 10$, we have found that there is no stable circular orbit for any values of $a$. These results suggest that stable circular orbits do not appear for $d \geq 6$ in general. On the other hand, how the sequences of circular orbits change by the separation $a$ for $d \geq 6$ is qualitatively the same as $d=5$. We expect that this property is also dimension independent in $d \geq 6$. It is worth noting that though the existence of a stable photon circular orbit is one of the specific geodesic structures in the 4D MP dihole spacetime, it is absent in $d \geq 5$.

As seen in the Schwarzschild-Tangherlini and MyersPerry black hole backgrounds, stable circular orbits tend not to appear for any dimensions of $d \geq 5$. This seems to be a property common to black objects with a large value of $d$. In fact, even in the MP dihole spacetime, we have found that stable circular orbits tend to be absent for any dimensions of $d \geq 6$. However, our results in 5D imply that there is a mechanism for the existence of stable circular orbits even in higher dimensions due to many-body effects, i.e., the existence of the center outside the horizon.

In contrast to the fact that the metric is analytic on the horizon of the 4D MP multi-black hole [44], the horizons of higher-dimensional ones are generally not smooth $[45,46]$. For $d=5$, the metric can be $C^{2}$ on the horizon but cannot be $C^{3}$ in general. For $d>5$, the metric is not even $C^{2}$ on the horizon, which leads to unavoidable curvature singularities. Hence, it should be noted that the results we have obtained in the case of $d \geq 6$ may include the effect of singularities.

For comparison with observations, we should discuss a higher-dimensional model of the universe, e.g., a higherdimensional black hole spacetime in which the extra dimensions are compactified. In particular, a 5D KaluzaKlein black hole spacetime with a twisted $S^{1}$ fiber behaves as a $5 \mathrm{D}$ spacetime near the horizon $\left(S^{3}\right.$ topology), whereas it effectively behaves as a 4D flat spacetime (i.e., a 4D flat spacetime with a compact dimension) in the asymptotic region $[47,48]$. From this point of view, the 5D KaluzaKlein black holes connect 4D spacetimes and 5D spacetimes as well as they have the feature of both 4D and 5D. Therefore, we expect that the size of the extra dimension should affect the sequence of stable circular orbits. This is an interesting issue for the future.

\section{ACKNOWLEDGMENTS}

This work was supported by the Grant-in-Aid for EarlyCareer Scientists [JSPS KAKENHI Grant No. JP19K14715 (T. I.)] and Grant-in-Aid for Scientific Research (C) [JSPS KAKENHI Grant No. JP17K05452 (S. T.)] from the Japan Society for the Promotion of Science. S. T. is also supported from Toyota Institute of Technology Fund for Research Promotion A.

\section{APPENDIX: UNSTABLE PHOTON CIRCULAR ORBIT IN THE EXTREMAL REISSNER-NORDSTRÖM SPACETIME}

We review an unstable photon circular orbit in the $d$-dimensional extremal Reissner-Nordström spacetime. The metric is given in isotropic coordinates by

$$
\begin{aligned}
\mathrm{ds}^{2}= & -\left(1+\frac{M}{r_{*}^{d-3}}\right)^{-2} \mathrm{~d} t^{2} \\
& +\left(1+\frac{M}{r_{*}^{d-3}}\right)^{2 /(d-3)}\left(\mathrm{d} r_{*}^{2}+r_{*}^{2} \mathrm{~d} \Omega_{d-2}^{2}\right),
\end{aligned}
$$

where $M$ is a mass parameter of the extremal black hole. This metric is derived by choosing $M_{+}=M_{-}=M / 2, a=0$, and $r_{*}=\sqrt{z^{2}+\rho^{2}}$ in Eqs. (4)-(6). In the Schwarzschild radial coordinate,

$$
r^{d-3}=r_{*}^{d-3}+M,
$$

the metric (A1) is written in the standard form

$$
\begin{aligned}
\mathrm{ds}^{2}= & -\left(1-\frac{M}{r^{d-3}}\right)^{2} \mathrm{~d} t^{2}+\left(1-\frac{M}{r^{d-3}}\right)^{-2} \mathrm{~d} r^{2} \\
& +r^{2}\left[\mathrm{~d} \theta^{2}+\sin ^{2} \theta\left(\mathrm{d} \phi^{2}+\sin ^{2} \phi \mathrm{d} \Omega_{d-4}^{2}\right)\right] .
\end{aligned}
$$

We consider the radial motion of a massless particle. Without loss of generality, we assume that a particle is confined to the equatorial plane, $\theta=\pi / 2$, and has an unique nonzero component of angular velocities $\dot{\phi} \neq 0$. Let us introduce $E=-\left(1-M / r^{d-3}\right)^{2} \dot{t}$, a conserved particle energy, and $L=r^{2} \dot{\phi}$, a conserved angular momentum. From the on-shell condition for null geodesics, $g_{\mu \nu} \dot{x}^{\mu} \dot{x}^{\nu}=0$, the radial equation in terms of these constants is given by

$$
\begin{aligned}
& \dot{r}^{2}+V=(E / L)^{2}, \\
& V=\left(\frac{1}{r}-\frac{M}{r^{d-2}}\right)^{2}
\end{aligned}
$$


where we have rescaled the affine parameter. The derivative of the potential function $V$ takes the form

$$
V^{\prime}=-\frac{2}{r^{2 d-3}}\left(r^{d-3}-M\right)\left[r^{d-3}-(d-2) M\right] .
$$

From $V^{\prime}=0$, we find that an extremum point of $V$ is located at

$$
r=\sqrt{d-3}(d-2) M
$$

Here exists an unstable circular orbit because $V$ has a local maximum point. In terms of $\rho$, the radius (A7) becomes

$$
\rho=\sqrt{d-3}(d-3) M .
$$

[1] D. C. Wilkins, Bound geodesics in the Kerr metric, Phys. Rev. D 5, 814 (1972).

[2] R. Emparan and H. S. Reall, Black holes in higher dimensions, Living Rev. Relativity 11, 6 (2008).

[3] F. R. Tangherlini, Schwarzschild field in $n$ dimensions and the dimensionality of space problem, Nuovo Cimento 27, 636 (1963).

[4] E. Hackmann, V. Kagramanova, J. Kunz, and C. Lammerzahl, Analytic solutions of the geodesic equation in higher dimensional static spherically symmetric space-times, Phys. Rev. D 78, 124018 (2008).

[5] V. P. Frolov and D. Stojkovic, Particle and light motion in a space-time of a five-dimensional rotating black hole, Phys. Rev. D 68, 064011 (2003).

[6] V. Kagramanova and S. Reimers, Analytic treatment of geodesics in five-dimensional Myers-Perry space-times, Phys. Rev. D 86, 084029 (2012).

[7] V. Diemer, J. Kunz, C. Lämmerzahl, and S. Reimers, Dynamics of test particles in the general five-dimensional Myers-Perry spacetime, Phys. Rev. D 89, 124026 (2014).

[8] V. Cardoso, A. S. Miranda, E. Berti, H. Witek, and V. T. Zanchin, Geodesic stability, Lyapunov exponents and quasinormal modes, Phys. Rev. D 79, 064016 (2009).

[9] T. Igata, Stable bound orbits in six-dimensional MyersPerry black holes, Phys. Rev. D 92, 024002 (2015).

[10] R. Emparan and R. C. Myers, Instability of ultra-spinning black holes, J. High Energy Phys. 09 (2003) 025.

[11] T. Delsate, J. V. Rocha, and R. Santarelli, Geodesic motion in equal angular momenta Myers-Perry-AdS spacetimes, Phys. Rev. D 92, 084028 (2015).

[12] S. Grunau, H. Neumann, and S. Reimers, Geodesic motion in the five-dimensional Myers-Perry-AdS spacetime, Phys. Rev. D 97, 044011 (2018).

[13] S. Hollands and S. Yazadjiev, Uniqueness theorem for 5-dimensional black holes with two axial Killing fields, Commun. Math. Phys. 283, 749 (2008).

[14] S. Hollands, J. Holland, and A. Ishibashi, Further restrictions on the topology of stationary black holes in five dimensions, Ann. Inst. Henri Poincaré 12, 279 (2011).

[15] M. 1. Cai and G. J. Galloway, On the topology and area of higher dimensional black holes, Classical Quantum Gravity 18, 2707 (2001).

[16] G. J. Galloway and R. Schoen, A generalization of Hawking's black hole topology theorem to higher dimensions, Commun. Math. Phys. 266, 571 (2006).
[17] R. Emparan and H.S. Reall, A Rotating Black Ring Solution in Five Dimensions, Phys. Rev. Lett. 88, 101101 (2002).

[18] J. Hoskisson, Particle motion in the rotating black ring metric, Phys. Rev. D 78, 064039 (2008).

[19] T. Igata, H. Ishihara, and Y. Takamori, Stable bound orbits around black rings, Phys. Rev. D 82, 101501 (2010).

[20] S. Grunau, V. Kagramanova, J. Kunz, and C. Lammerzahl, Geodesic motion in the singly spinning black ring spacetime, Phys. Rev. D 86, 104002 (2012).

[21] T. Igata, H. Ishihara, and Y. Takamori, Stable bound orbits of massless particles around a black ring, Phys. Rev. D 87, 104005 (2013).

[22] G. W. Gibbons and S. W. Hawking, Classification of gravitational instanton symmetries, Commun. Math. Phys. 66, 291 (1979).

[23] T. Igata, Particle dynamics in the Newtonian potential sourced by a homogeneous circular ring, Phys. Rev. D 101, 124064 (2020).

[24] T. Igata, Chaotic particle motion around a homogeneous circular ring, Phys. Rev. D 102, 044019 (2020).

[25] H. K. Kunduri and J. Lucietti, Supersymmetric Black Holes with Lens-Space Topology, Phys. Rev. Lett. 113, 211101 (2014).

[26] S. Tomizawa and M. Nozawa, Supersymmetric black lenses in five dimensions, Phys. Rev. D 94, 044037 (2016).

[27] S. Tomizawa and T. Igata, Stable bound orbits around a supersymmetric black lens, Phys. Rev. D 100, 124031 (2019).

[28] S. Majumdar, A class of exact solutions of Einstein's field equations, Phys. Rev. 72, 390 (1947).

[29] A. Papaetrou, A static solution of the equations of the gravitational field for an arbitrary charge distribution, Proc. R. Irish Acad. A 51, 191 (1947).

[30] R. C. Myers, Higher dimensional black holes in compactified space-times, Phys. Rev. D 35, 455 (1987).

[31] S. Chandrasekhar, The two center problem in general relativity: The scattering of radiation by two extreme ReissnerNordström black holes, Proc. R. Soc. A 421, 227 (1989).

[32] G. Contopoulos, Periodic orbits and chaos around two fixed black holes, Proc. R. Soc. A 431, 183 (1990).

[33] A. Wunsch, T. Müller, D. Weiskopf, and G. Wunner, Circular orbits in the extreme Reissner-Nordström dihole metric, Phys. Rev. D 87, 024007 (2013). 
[34] S. R. Dolan and J. O. Shipley, Stable photon orbits in stationary axisymmetric electrovacuum spacetimes, Phys. Rev. D 94, 044038 (2016).

[35] T. Ono, T. Suzuki, and H. Asada, Nonradial stability of marginal stable circular orbits in stationary axisymmetric spacetimes, Phys. Rev. D 94, 064042 (2016).

[36] T. Assumpcao, V. Cardoso, A. Ishibashi, M. Richartz, and M. Zilhao, Black hole binaries: Ergoregions, photon surfaces, wave scattering, and quasinormal modes, Phys. Rev. D 98, 064036 (2018).

[37] K. Nakashi and T. Igata, Innermost stable circular orbits in the Majumdar-Papapetrou dihole spacetime, Phys. Rev. D 99, 124033 (2019).

[38] K. Nakashi and T. Igata, Effect of a second compact object on stable circular orbits, Phys. Rev. D 100, 104006 (2019).

[39] G. Contopoulos, Periodic orbits and chaos around two fixed black holes. II, Proc. R. Soc. A 435, 551 (1991).

[40] J. Shipley and S. R. Dolan, Binary black hole shadows, chaotic scattering and the Cantor set, Classical Quantum Gravity 33, 175001 (2016).
[41] D. Nitta, T. Chiba, and N. Sugiyama, Shadows of colliding black holes, Phys. Rev. D 84, 063008 (2011).

[42] M. Patil, P. Mishra, and D. Narasimha, Curious case of gravitational lensing by binary black holes: A tale of two photon spheres, new relativistic images and caustics, Phys. Rev. D 95, 024026 (2017).

[43] W. Hanan and E. Radu, Chaotic motion in multi-black hole spacetimes and holographic screens, Mod. Phys. Lett. A 22, 399 (2007).

[44] J. Hartle and S. Hawking, Solutions of the Einstein-Maxwell equations with many black holes, Commun. Math. Phys. 26, 87 (1972).

[45] D. L. Welch, On the smoothness of the horizons of multiblack hole solutions, Phys. Rev. D 52, 985 (1995).

[46] G. N. Candlish and H. S. Reall, On the smoothness of static multi-black hole solutions of higher-dimensional EinsteinMaxwell theory, Classical Quantum Gravity 24, 6025 (2007).

[47] S. Tomizawa and H. Ishihara, Exact solutions of higher dimensional black holes, Prog. Theor. Phys. Suppl. 189, 7 (2011).

[48] S. Tomizawa, Kaluza-Klein black lens in five dimensions, Phys. Rev. D 98, 024012 (2018). 